\title{
Influence de la température sur la croissance in vitro d'hyphomycètes entomopathogènes
}

\author{
J Fargues 1, NK Maniania 2, JC Delmas 1, 3, N Smits 1 \\ 1 INRA, station de recherche de lutte biologique, La Minière, 78285 Guyancourt Cedex, France; \\ 2 International Center of Insect Physiology and Ecology, PO Box 30772, Nairobi, Kenya; \\ 3 Université Paris 7, UFR biologie et sciences de la nature, 2 Place Jussieu, 75251 Paris Cedex 05, France.
}

(Reçu le 16 décembre 1991; accepté le 22 avril 1992)

\begin{abstract}
Résumé - L'influence de la température sur la croissance in vitro des principales espèces d'hyphomycètes entomopathogènes a été étudiée avec 31 isolats fongiques : 3 souches de Beauveria bassiana (Balsamo) Vuillemin, 3 souches de $B$ brongniartii (= tenella) (Saccardo) Petch, 8 souches de Metarhizium anisopliae (Metschnikoff) Sorokin, 1 souche de $M$ flavoviride Gams et Rozsypal, 6 souches de Nomuraea rileyi (Farlow) Samson et 10 souches de Paecilomyces fumosoroseus (Wize) Brown et Smith. L'analyse des vitesses de croissance radiale à 10 niveaux de température compris entre 8 et $37^{\circ} \mathrm{C}$ montre une variabilité à la fois inter- et intraspécifique, liée à l'origine géoclimatique des isolats fongiques. Les champignons provenant de zones subtropicales humides sont relativement thermophiles avec des gammes d'activité significative de $11^{\circ}$ et $32^{\circ} \mathrm{C}$ (isolats de $\mathrm{N}$ rileyi) ou $35^{\circ} \mathrm{C}$ (isolats de $\mathrm{M}$ anisopliae). Inversement, les souches originaires de zones tempérées et surtout du sol de ces régions (isolats issus d'insectes vivants dans le sol ou d'échantillons de sol) présentent une croissance non négligeable aux basses températures $\left(8^{\circ} \mathrm{C}\right.$ pour les isolats de $B$ bassiana et ceux de $B$ brongniarti). Néanmoins, l'optimum thermique de croissance de la plupart des isolats (26 sur 31$)$ se situe à $25^{\circ} \mathrm{C}$. Enfin, si certains isolats ont une gamme thermique d'activité végétative relativement étroite (chez $B$ brongniartii et $P$ fumosoroseus) d'autres sont très thermotolérants (la plupart des isolats de $M$ anisopliae). La tolérance thermique des champignons entomopathogènes apparaît donc comme un critère de sélection des isolats fongiques candidats à la lutte microbiologique mieux adaptés au biotope de l'insecte-hôte visé.
\end{abstract}

température / croissance in vitro / hyphomycète entomopathogène / sélection / lutte biologique

Summary - Influence of temperature on in vitro growth of entomopathogenic hyphomycetes. Thirty-one isolates of 6 entomopathogenic hyphomycetes, Beauveria bassiana (Balsamo) Vuillemin, B brongniartii (= tenella) (Saccardo) Petch, Metarhizium anisopliae (Metschnikoff) Sorokin, M flavoviride Gams et Rozsypal, Nomuraea rileyi (Farlow) Samsom and Paecilomyces fumosoroseus (Wize) Brown et Smith, were grown in vitro to determine growth rates and ranges of growth at 10 temperatures from 8 to $37^{\circ} \mathrm{C}$ in the dark. Radial growth of surface colonies on semisynthetic nutrient agar was recorded by daily measurement of 2 cardinal diameters. Because radial measurements (from day 3 to 12) of surface colonies for each temperature fit a linear model $(y=v t+b)$, growth rates $\left(v\right.$ in $m m x$ day $\left.{ }^{-1}\right)$ were used as the main growth parameter to evaluate the influence of temperature. The fungal isolates under study exhibited a wide diversity of responses to temperature according to their provenance. Isolates originating from tropical areas showed a remarkable growth at $35^{\circ} \mathrm{C}$ (eg, $\mathrm{M}$ anisopliae isolates). By contrast, isolates originating from soil-inhabiting insects of temperate areas (eg B brongniartii isolates) exhibited high growth rates at $8{ }^{\circ} \mathrm{C}$. However, the optimal growth was found at $25^{\circ} \mathrm{C}$ for 26 isolates. Upper temperature limits of growth varied from $28-37^{\circ} \mathrm{C}$ according to both fungal species and isolates. $\mathrm{M}$ anisopliae isolates exhibited an almost ideal combination of high growth rates and in particular a wide temperature range $\left(8-11^{\circ}\right.$ to $35-37^{\circ} \mathrm{C}$ ); only one $\mathrm{B}$ bassiana isolate showed similar responses to temperature stimuli. Although the optimal temperature for fungal growth is not necessarily the same as that for fungal infection in insects, temperature ranges established according to in vitro growth data might be used for selecting fungal candidates for microbial control.

temperature / in vitro growth / entomopathogenic hyphomycete / selection / microbial control 


\section{INTRODUCTION}

La température est un des principaux facteurs du milieu qui limitent le développement des mycoses dans les populations naturelles d'insectes (Carruthers et al, 1985; Carruthers et Soper, 1987; Benz, 1987). Aussi, en lutte microbiologique, il convient de rechercher des champignons entomopathogènes dont la gamme de tolérance à l'égard de la température soit compatible avec les conditions climatiques de l'écosystème visé (Ferron et al, 1991). Ainsi, pour lutter contre les curculionides en zone tempérée, Doberski (1981) et Soares et al (1983) ont sélectionné des isolats fongiques dont l'activité pathogène se maintient à des températures inférieures à 15 ${ }^{\circ} \mathrm{C}$. À l'opposé, Ignoffo et al (1976a) et Mohammed et al (1977) ont étudié l'effet des températures supérieures à $25^{\circ} \mathrm{C}$ sur diverses souches de Nomuraea rileyi (Farlow) Samson inféodées aux noctuelles de l'agroécosystème soja dans le Sud-Est des États-Unis d'Amérique (Ignoffo et al, 1976b; Ignoffo, 1981).

L'influence de la température sur la croissance in vitro des principales espèces d'hyphomycètes entomopathogènes, Beauveria bassiana (Balsamo) Vuillemin, $B$ brongniartii (Saccardo) Petch, Metarhizium anisopliae (Metschnikoff) Sorokin, $M$ flavoviride Gams et Rozsypal, $N$ rileyi et Paecilomyces fumosoroseus (Wize) Brown et Smith, a été étudiée à 10 niveaux constants compris entre $8^{\circ}$ et $37^{\circ} \mathrm{C}$. Trente et un isolats fongiques issus de différentes espèces hôtes, d'origines géoclimatiques diverses, ont été retenus en raison de leur activité pathogène à l'égard d'insectes d'intérêt agronomique tels que Leptinotarsa decemlineata Say (Chrysomelidae) (Fargues, 1972; 1976), divers Scarabaeidae (Ferron, 1967; Ferron et al, 1972; Fargues et al, 1976), des Curculionidae (Soares et al, 1983; Poprawski et al, 1985), Plutella maculipennis Curtis (Hyponomeutidae) (Robert et Marchal, 1980) ou Ostrinia nubilalis Hübner (Pyralidae) (Riba et al, 1983).

\section{MATÉRIELS ET MÉTHODES}

Les essais ont été conduits avec 3 souches de $B$ bassiana $(B b), 3$ souches de $B$ brongniartii $(B b r), 8$ souches de $M$ anisopliae (Ma), 1 souche de $M$ flavoviride (Mf), $10 P$ fumosoroseus (Pfr) et $8 \mathrm{~N}$ rileyi $(\mathrm{Nr})$.

Ces microrganismes, conservés à la mycothèque INRA de la station de recherches de lutte biologique du centre de Versailles, ont des origines géographi- ques dont la diversité est variable selon les espèces considérées (tableau I). Ainsi, les souches de $M$ anisopliae proviennent de France, de Madagascar, de la Guadeloupe, etc., alors que toutes celles de $P$ fumosoroseus proviennent de France. Par ailleurs, toutes ont été isolées à partir de cadavres d'insectes atteints de mycose, à l'exception de la souche $B b r 74$, issue d'un échantillon de sol (tableau I). Les cultures ont été faites sur milieu semi-synthétique gélosé (en g. $\mathrm{I}^{-1}$ : $0,39 \quad \mathrm{KH}_{2} \mathrm{PO}_{4}, \quad 1,42 \quad \mathrm{Na}_{2} \mathrm{HPO}_{4} .12 \mathrm{H}_{2} \mathrm{O}, \quad 0,60$ $\mathrm{MgSO}_{4} .7 \mathrm{H}_{2} \mathrm{O}, 1,00 \mathrm{KCl}, 10$ glucose, $0,70 \mathrm{NH}_{4} \mathrm{HO}_{3}, 5$ extrait de levure, 20 agar) à $25 \pm 1^{\circ} \mathrm{C}$ pendant 3 semaines avant de recueillir les inoculums sporaux.

L'influence de la température sur l'activité végétative des différents isolats fongiques a été analysée en mesurant la croissance radiale in vitro d'explantats calibrés (Rapilly, 1968). Des rondelles de culture non sporulée ( $3 \mathrm{j}$ après ensemencement) ont été prélevées à l'aide d'un emporte-pièce et transférées sur milieu nutritif stérile à raison d'un explant $(6 \mathrm{~mm}$ de diamètre) par boîte de Petri (50 mm de diamètre). Pour chaque température, 4 boîtes de Petri ont été placées en atmosphère saturée en humidité et à l'abri de la lumière. Dix niveaux de température ont été retenus : 8 , $11,15,20,25,28,30,32,35$ et $37^{\circ} \mathrm{C} \pm 1$. Deux axes orthogonaux ayant été tracés préalablement sur le fond de chaque boîte de Petri, les 2 diamètres de la colonie ont été mesurés quotidiennement suivant ces repères. Par isolat et par température, 4 séries de 2 mesures ont été effectuées quotidiennement. Chacune de ces séries de mesures a fait l'objet d'une analyse de régression $(y=v t+b)$ dans le but d'utiliser la vitesse de croissance (pente de la droite) comme critère d'estimation de l'effet de la température. Les vitesses de croissance radiale ont été soumises à l'analyse de variance à 2 facteurs : température (à 8 niveaux) et isolat fongique (nombre de niveaux variable suivant l'espèce entomopathogène considérée) (effets significatifs aux seuils de $5 \%$, $1 \%^{\star *}$, ou $\left.0,1 \%{ }^{\star \star \star}\right)$.

\section{RÉSULTATS}

Dans les conditions expérimentales retenues, la croissance radiale des divers isolats fongiques s'est avérée linéaire entre le $3^{e}$ et le $12^{e} j$ d'incubation, avec des coefficients de corrélation compris entre 0,994 et 0,999 aux températures favorables $\left(20^{\circ}-30^{\circ} \mathrm{C}\right)$ et entre 0,860 et 0,990 aux températures marginales $\left(<15^{\circ} \mathrm{C}\right.$ et $\left.>32^{\circ} \mathrm{C}\right)$. Les pentes des droites ainsi définies étant des constantes, les vitesses de croissance de chaque champignon, à chaque niveau thermique étudié, ont été présentées sous forme de données moyennes exprimées en $\mathrm{mm} . \mathrm{j}^{-1}$ (tableaux II et III) et elles ont été figurées en valeur relative (\%) par rapport à la vitesse maximale $\left(v_{\max }=\right.$ $100 \%$ ) au sein de chacun des isolats de $B$ bas- 
Tableau I. Origine des souches fongiques entomopathogènes étudiées.

\begin{tabular}{|c|c|c|c|}
\hline \multirow{2}{*}{$\begin{array}{l}\text { Espèce } \\
\text { et isolat }\end{array}$} & \multicolumn{2}{|l|}{ Origine } & \multirow[b]{2}{*}{ Lieu d'isolement } \\
\hline & Espèce hôte d'origine & d'isolement & \\
\hline \multicolumn{4}{|c|}{ Beauveria bassiana } \\
\hline$B b 18$ & Epinotia cedricida Diak (Tortricidae) & 1968 & France (Haute-Alpes) \\
\hline$B b 93$ & Laspeyresia pomonella L (Tortricidae) & 1974 & Autriche \\
\hline$B b 147$ & Ostrinia nubilalis Hübner (Pyralidae) & 1978 & France (Beauce) \\
\hline \multicolumn{4}{|c|}{$B$ brongniartii } \\
\hline Bbr 6 & Melonlontha melolontha L (Scarabaeidae) & 1964 & France (Sarthe) \\
\hline$B b r 74$ & Echantillon de sol & 1976 & France (Doubs) \\
\hline$B b r 76$ & $M$ melolontha & 1976 & France (Cantal) \\
\hline \multicolumn{4}{|c|}{ Metarhizium anisopliae } \\
\hline Ma 32 & Cetonia aurata L (Scarabaeidae) & 1967 & France \\
\hline Ma 51 & Oryctes rhinoceros L (Scarabaeidae) & 1971 & Madagascar \\
\hline Ma 78 & Hoplochelus rhizotrogoides Blanch (Scarabaeidae) & 1973 & Madagascar \\
\hline Ma 84 & Cosmopolites sordidus Germar (Curculionidae) & 1974 & Guadeloupe \\
\hline Ma 115 & Mahanarva posticata Stål (Miridae) & 1978 & Brésil \\
\hline Ma 120 & Moustique non identifié & 1978 & États Unis \\
\hline Ma 131 & Mposticata & 1980 & Brésil \\
\hline Ma 139 & O nubilalis & 1980 & France (Beauce) \\
\hline \multicolumn{4}{|c|}{$M$ flavoviride } \\
\hline$M f$ & Otiorhynchus suloatus Fabr (Curculionidae) & 1974 & France (Bretagne) \\
\hline \multicolumn{4}{|c|}{ Nomuraea rileyi } \\
\hline Nr 4 & Anticarsia gemmatalis Hübner (Noctuidae) & 1974 & États Unis (Caroline du Sud) \\
\hline Nr5 & A gemmatalis & 1974 & États Unis (Floride) \\
\hline Nr6 & A gemmatalis & 1976 & États Unis (Missouri) \\
\hline$N r 7$ & Bombyx mori L (Bombycidae) & 1979 & Japon \\
\hline Nr 8 & Spilosoma sp F23 (Arctiidae) & 1979 & Japon \\
\hline Nr9 & A gemmatalis & 1980 & Brésil \\
\hline \multicolumn{4}{|c|}{ Paecilomyces fumosoroseus } \\
\hline Pfr 4 & Mamestra brassicae L (Noctuidae) & 1972 & France (lle-de-France) \\
\hline Pfr 13 & Lycophotia sp (Noctuidae) & 1972 & France (Provence) \\
\hline Pfr 24 & Chilo supressalis Walker (Pyralidae) & 1974 & France (lle-de-France) \\
\hline Pfr 28 & $M$ brassicae & 1975 & France (lle-de-France) \\
\hline Pfr 32 & Socita ipsilon Hübner (Noctuidae) & 1976 & France (lle-de-France) \\
\hline Pfr 35 & $M$ brassicae & 1976 & France (lle-de-France) \\
\hline Pfr 36 & Scotia segetum Schiffermüller (Noctuidae) & 1976 & France (Ile-de-France) \\
\hline Pfr 39 & S segetum & 1976 & France (lle-de-France) \\
\hline Pfr 40 & O nubilalis & 1977 & France (Béarn) \\
\hline Pfr 45 & Pieris brassicae L (Pieridae) & 1978 & France (lle-de-France) \\
\hline
\end{tabular}

siana et $B$ brongniartii (fig 1), de $M$ anisopliae et $M$ flavoviride (fig 2), de $N$ rileyi (fig 3 ) et de $P$ fumosoroseus (fig 4).

L'optimum de croissance de la plupart des isolats (25 sur 31) des 6 espèces fongiques étudiées se situe à $25^{\circ} \mathrm{C}$. Leurs vitesses maximales de croissance radiale sont comprises entre 3,0 et $4,5 \mathrm{~mm} . \mathrm{j}^{-1}$, à l'exception de celles des isolats de $N$ rileyi qui varient de 0,52 à $1,25 \mathrm{~mm}^{-1}$.
D'une manière générale, la gamme de températures favorables à la croissance fongique est relativement homogène pour chacune des espèces entomopathogènes (tableaux II et III). Les isolats les mieux adaptés aux faibles températures sont ceux de $B$ bassiana ( 8 à $32^{\circ} \mathrm{C}$ ) (fig 1) et de $B$ brongniartii ( 8 à $30^{\circ} \mathrm{C}$ ), ces 2 espèces étant les seules dont la croissance des souches n'est pas inhibée à $8{ }^{\circ} \mathrm{C}$ (fig 1). En dessous de 


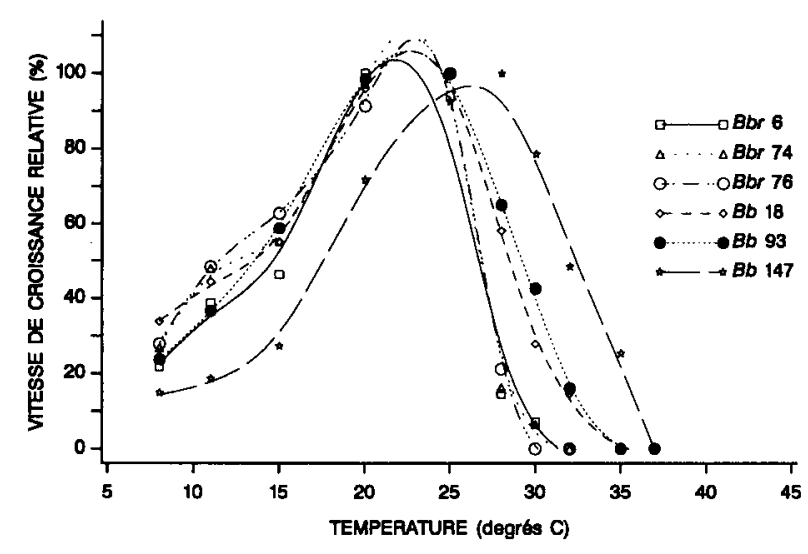

Fig 1. Influence de la température sur la croissance linéaire de diverses souches de Beauveria bassiana $(\mathrm{Bb})$ et de $B$ brongniartii $(\mathrm{Bbr})$ : vitesses exprimées en valeur relative par rapport à la vitesse de croissance maximale de chaque isolat.

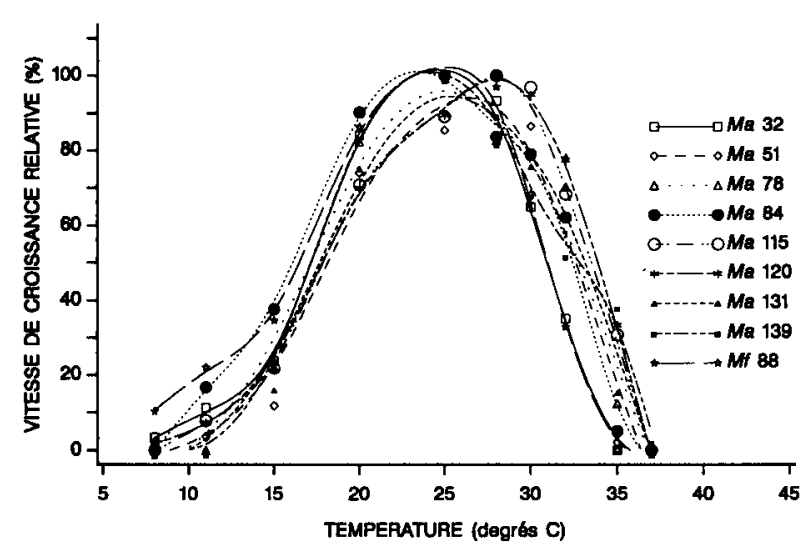

Fig 2. Influence de la température sur la croissance linéaire de diverses souches de Metarhizium anisopliae (Ma) et de $\mathrm{M}$ flavoviride (Mf) : vitesses exprimées en valeur relative par rapport à la vitesse de croissance maximale de chaque isolat.

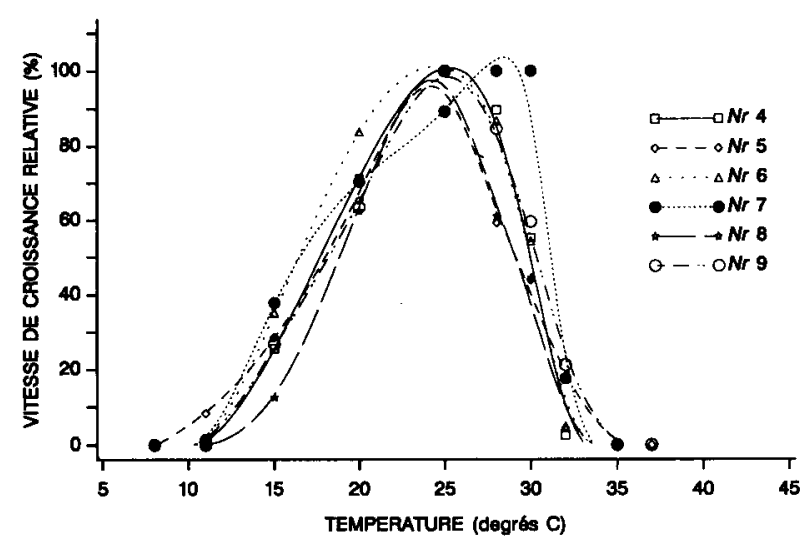

Fig 3. Influence de la température sur la croissance linéaire de diverses souches de Nomuraea rileyi $(\mathrm{Nr})$ : vitesses exprimées en valeur relative par rapport à la vitesse de croissance maximale de chaque isolat.

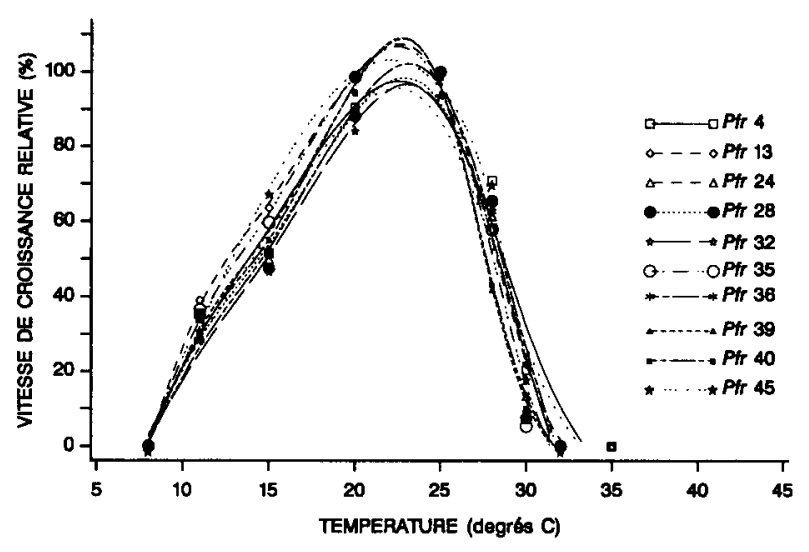

Fig 4. Influence de la température sur la croissance linéaire de diverses souches de Paecilomyces fumosoroseus (Pfr) : vitesses exprimées en valeur relative par rapport à la vitesse de croissance maximale de chaque isolat.

$11^{\circ} \mathrm{C}$, ce sont les isolats de $N$ rileyi qui sont les moins bien adaptés $\left(15-32{ }^{\circ} \mathrm{C}\right.$ ) (fig 3 ). Les isolats de $P$ fumosoroseus sont relativement peu tolérants $\left(11-30^{\circ} \mathrm{C}\right.$ ) (fig 4). Si l'on considère l'effet des températures maximales, on peut noter que $P$ fumosoroseus est l'espèce pour laquelle la température d'inhibition de la croissance pour la majorité des souches est la moins élevée $\left(32^{\circ} \mathrm{C}\right)$. De plus, cette espèce est celle pour laquelle les courbes de vitesse de croissance des différentes souches sont les plus homogènes (fig 4). Les isolats de $M$ anisopliae présentent les gammes de température de croissance les plus larges $\left(8 / 11-35 / 37^{\circ} \mathrm{C}\right)$ (fig 2).

La variabilité infraspécifique de la vitesse de croissance des champignons étudiés résulte à la fois de l'aptitude de chaque isolat à croître sur le milieu nutritif semi-synthétique de référence et de sa réponse spécifique aux stimuli thermiques (effets «souche» et «interaction» très hautement significatifs) (tableaux II et III). Ainsi, au-delà des différences observées entre espèces fongiques, certains isolats présentent des comportements particuliers à l'égard de la température. L'optimum thermique de croissance des isolats $B b r 6$ et Pfr 45 est de $20^{\circ} \mathrm{C}$, alors que celui des isolats Bb 147, Ma 51, Ma 115, Ma 120 et $\mathrm{Nr} 7$ est de $28^{\circ} \mathrm{C}$. Aux faibles températures, l'isolat $B b 18$ se distingue des autres souches de Beauveria sp par sa vitesse de croissance élevée à $8^{\circ} \mathrm{C}$ (vitesse relative par rapport à l'optimum de $34 \%$ ) (fig 1). En revanche, d'autres isolats tels que $\mathrm{Ma}$ 115 (vitesse relative de $31 \%-35{ }^{\circ} \mathrm{C}$ ), Ma 120 $\left(33 \%\right.$ à $\left.35^{\circ} \mathrm{C}\right)$, Ma $139\left(39 \%-35^{\circ} \mathrm{C}\right)$ et $\mathrm{Bb} 147$ $\left(25 \%\right.$ à $\left.35^{\circ} \mathrm{C}\right)$ sont particulièrement bien adaptés aux fortes températures (figs 1,2 ). 


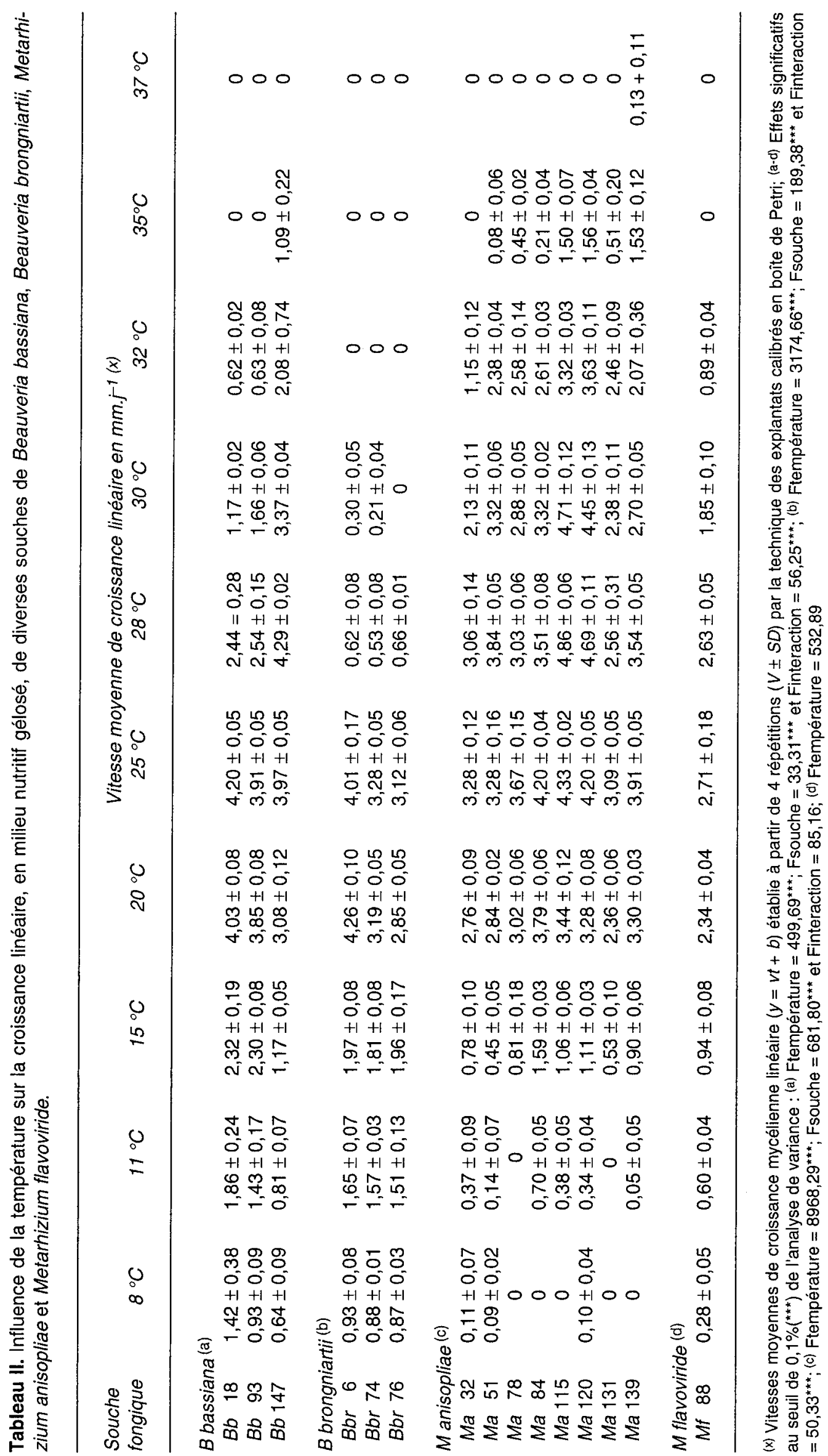


Tableau III. Influence de la température sur la croissance linéaire, en milieu nutritif gélosé, de diverses souches de Nomuraea rileyi et de Paecilomyces fumosoroseus.

Souche

fongique $8^{\circ} \mathrm{C} \quad 11^{\circ} \mathrm{C}$
Vitesse moyenne de croissance linéaire en $m m \cdot \dot{\zeta}^{1(x)}$

$\begin{array}{lllll}15^{\circ} \mathrm{C} & 20{ }^{\circ} \mathrm{C} & 25^{\circ} \mathrm{C} & 28^{\circ} \mathrm{C} & 30{ }^{\circ} \mathrm{C}\end{array}$

$32{ }^{\circ} \mathrm{C} \quad 35^{\circ} \mathrm{C}$
N rileyi (a)

$\begin{array}{cccc}\text { Nr4 } & 0 & 0 & 0,32 \pm 0,09 \\ \text { Nr5 } & 0 & 0,08 \pm 0,04 & 2,28 \pm 0,03 \\ \text { Nr6 } & 0 & 0 & 0,37 \pm 0,05 \\ \text { Nr7 } & 0 & 0,01 \pm 0,01 & 0,28 \pm 0,05 \\ \text { Nr8 } & 0 & 0 & 0,09 \pm 0,05 \\ \text { Nr9 } & 0 & 0 & 0,14 \pm 0,03\end{array}$

$P$ fumosoroseus (b)

$\begin{array}{lll}\text { Pfr 4 } & 0 & 1,46 \pm 0,09 \\ \text { Pfr 13 } & 0 & 1,38 \pm 0,11 \\ \text { Pfr 24 } & 0 & 1,38 \pm 0,06 \\ \text { Pfr 28 } & 0 & 1,41 \pm 0,02 \\ \text { Pfr 32 } & 0 & 1,27 \pm 0,19 \\ \text { Pfr 35 } & 0 & 1,34 \pm 0,26 \\ \text { Pfr 36 } & 0 & 1,38 \pm 0,04 \\ \text { Pfr 39 } & 0 & 1,35 \pm 0,21 \\ \text { Pfr 40 } & 0 & 1,51 \pm 0,02 \\ \text { Pfr 45 } & 0 & 1,11 \pm 0,07\end{array}$

$2,12 \pm 0,04$

$2,25 \pm 0,08$

$2,03 \pm 0,06$

$1,92 \pm 0,06$

$2,05 \pm 0,04$

$2,19 \pm 0,02$

$2,49 \pm 0,04$

$2,39 \pm 0,10$

$2,63 \pm 0,04$

$2,37 \pm 0,08$
$0,89 \pm 0,04$

$0,64 \pm 0,04$

$0,88 \pm 0,02$

$0,52 \pm 0,04$

$0,45 \pm 0,08$

$0,33 \pm 0,06$

\section{$3,72 \pm 0,08$}

$3,50 \pm 0,06$

$3,61 \pm 0,12$

$3,56 \pm 0,06$

$3,71 \pm 0,03$

$3,62 \pm 0,05$

$4,37 \pm 0,05$

$4,04 \pm 0,11$

$4,67+0,06$

$3,46 \pm 0,35$
$1,25 \pm 0,07$

$0,98 \pm 0,04$

$1,05 \pm 0,01$

$0,66 \pm 0,03$

$0,72 \pm 0,18$

$0,52 \pm 0,04$
$1,12 \pm 0,10$

$0,58 \pm 0,02$

$0,91 \pm 0,04$

$0,74 \pm 0,16$

$0,44 \pm 0,05$

$0,44 \pm 0,06$

\section{$4,11 \pm 0,04$}

$3,54 \pm 0,22$

$4,06 \pm 0,06$

$4,04 \pm 0,03$

$4,41 \pm 0,07$

$3,67 \pm 0,02$

$4,89 \pm 0,05$

$4,22 \pm 0,05$

$4,85 \pm 0,10$

$3,29 \pm 0,02$

\section{$2,91 \pm 0,16$}

$2,05 \pm 0,16$

$2,49 \pm 0,32$

$2,64 \pm 0,17$

$2,82 \pm 0,12$

$2,12 \pm 0,15$

$3,06 \pm 0,30$

$1,82 \pm 0,08$

$2,17 \pm 0,15$

$2,46 \pm 0,15$
$0,69 \pm 0,03$

$0,43 \pm 0,03$

$0,57 \pm 0,05$

$0,74 \pm 0,02$

$0,32 \pm 0,05$

$0,31 \pm 0,01$

$0,03 \pm 0,03$

$0,18 \pm 0,01$

$0,05 \pm 0,06$

$0,13 \pm 0,05$

$0,33 \pm 0,04$

$0,11 \pm 0,04$
0

0

0

0

0

(x) Vitesses moyennes de croissance mycélienne linéaire $(y=v t+b)$ établie à partir de 4 répétitions $(V \pm S D)$ par la technique des explants calibrés en boite de Petri; ${ }^{(a-d)}$ Effets significatifs au seuil de $0,1 \%\left({ }^{\star \star \star}\right)$ de l'analyse de variance : (a) Ftempérature $=766,89^{* \star *}$; Fsouche $=161,66^{\star \star *}$ et Finteraction $=24,68^{\star \star *}$; (b) Ftempérature $=4484,13^{\star \star \star}$; Fsouche $=72,64^{\star \star *}$ et Finteraction $=20,64^{\star * *}$

Enfin, certains isolats présentent une gamme thermique d'activité particulièrement large, notamment $\mathrm{Bb} 147\left(15 \%\right.$ à $8{ }^{\circ} \mathrm{C}$ et $25,4 \%$ à $\left.35^{\circ} \mathrm{C}\right)$ (fig 1) et $\mathrm{Ma} 120\left(2,1 \%\right.$ à $8{ }^{\circ} \mathrm{C}$ et $33,3 \%$ à $\left.35^{\circ} \mathrm{C}\right)$ (fig 2).

\section{DISCUSSION}

Les 6 espèces d'hyphomycètes entomopathogènes étudiées sont, comme la plupart des champignons, des microrganismes mésophiles (Gray et Williams, 1971; Deacon, 1980) puisque leur gamme de croissance couvre des températures modérées $\left(8-30^{\circ} \mathrm{C}\right.$ pour $\mathrm{B}$ brongniartii et 11-35 ${ }^{\circ} \mathrm{C}$ pour $M$ anisopliae), dont l'optimum est compris entre 20 et $28^{\circ} \mathrm{C}\left(25^{\circ} \mathrm{C}\right.$ pour la majorité des isolats). Ces données confirment que les températures les plus favorables aux champignons entomopathogènes se trouvent entre 15 et $30^{\circ} \mathrm{C}$ (Roberts et Campbell, 1977; Hall et Papierok, 1982; Ferron et al, 1991).

Les essais de croissance in vitro des 31 isolats fongiques ont révélé une variabilité inter- et intraspécifique de la réponse de ces champi- gnons aux stimuli thermiques. Les différences observées concernent essentiellement les niveaux des seuils minimaux et maximaux ainsi que l'étendue des gammes de température d'activité.

Les résultats montrent une relation étroite entre les seuils thermiques minimaux et maximaux de croissance et les conditions climatiques de l'environnement d'origine du champignon. Ainsi, les isolats les plus thermophiles proviennent des zones subtropicales humides de Madagascar ( $M a 51$ et $M a 78$ ) ou d'Amérique (Ma 84, Ma 115, Ma 120, Ma 131; Nr 4, Nr 5, Nr6 et $\mathrm{Nr}$ 9) (tableau I). Les isolats Bb 147 et Ma 139, dont les croissances sont encore significatives à 35 et à $37{ }^{\circ} \mathrm{C}$, respectivement, apparaissent comme des exceptions, puisqu'ils ont été recueillis en région parisienne. Toutefois, l'origine méridionale récente de leur hôte d'isolement, Ostrinia nubilalis Hübner (Riba et al, 1983) peut être évoquée comme hypothèse explicative. Inversement, l'adaptation aux faibles températures des isolats de $B$ bassiana, de $B$ brongniartii et de $M$ flavoviride est à rapprocher de leur origine tempérée dans des sites en altitude (Alpes françaises pour $B b 18$ et autrichiennes pour $B b$ 93) et/ou dans le 
sol ( $B b r 6, B b r 74$ et $B b r 76$ ainsi que $M f 88$ ). Dans le même ordre d'idée, Arkhipova (1965, citée par Roberts et Campbell, 1977) rapporte que les souches de $B$ bassiana, isolées au Nord de la Russie, sont moins thermophiles que les isolats recueillis dans le Sud de l'Union Soviétique.

En général, les optimums thermiques de croissance, déterminés par l'analyse macroscopique du développement de colonies fongiques in vitro, sont étroitement corrélés avec les optimums de germination et de sporulation (Roberts et Campbell, 1977; Hall et Papierok, 1982). Néanmoins, certains auteurs ont observé la germination des conidiospores de divers hyphomycètes à des températures inférieures $\left(5-8^{\circ} \mathrm{C}\right)$ aux seuils minimaux de croissance des souches étudiées (Ignoffo et al, 1976a; Kish et Allen, 1978). En dehors de quelques exceptions (Fargues, 1972; Fargues et Rodriguez-Rueda, 1980; Maniania et Fargues, 1992) la plupart des travaux ont aussi souligné la concordance entre l'optimum thermique de l'activité végétative du champignon et celui de l'infection fongique sur l'insecte hôte (Hall et Papierok, 1982; Ferron, 1985).

Certains isolats fongiques, tels que $B b 147$, Ma 51, Ma 120 ou Ma 139, ont présenté des gammes thermiques de croissance particulièrement étendues $\left(8-35^{\circ} \mathrm{C}\right.$ ou $\left.11-37^{\circ} \mathrm{C}\right)$ ce qui leur confère une remarquable adaptabilité. En effet, bien que les mécanismes de thermorégulation permettent aux insectes hôtes d'échapper en partie aux effets des températures extrêmes du milieu, les conditions thermiques de leurs habitats varient considérablement suivant l'espèce et l'agroécosystème considéré (Heinrich, 1981).

La tolérance thermique des isolats entomopathogènes apparaît donc bien comme un critère de sélection des hyphomycètes candidats à la lutte microbiologique (Goral, 1973; Isaenko et al, 1974; III'Icheva et al, 1976). La variabilité de ce caractère permet en outre d'envisager l'amélioration des potentialités des souches les plus pathogènes par recombinaison (Yurchenko et al, 1974; Paris, 1977; Al-Aidroos, 1980; Messias et Azevado, 1980; Riba et al, 1984; Riba et Ravelojoana, 1984) avec des isolats sélectionnés pour leur tolérance à l'égard des conditions climatiques des agrosystèmes infestés par l'insecte cible.

\section{REMERCIEMENTS}

Les auteurs remercient $H$ Vermeil de Conchard pour sa collaboration technique ainsi que $P$ Cassier de
I'Université Paris VI et B Papierok de I'Institut Pasteur pour leur révision critique du manuscrit.

\section{RÉFÉRENCES}

Al-Aidroos K (1980) Demonstration of a parasexual cycle in the entomopathogenic fungus Metarhizium anisopliae. Can J Genet Cytol 22, 771-775

Benz G (1987) Environment. In: Epizootiology of insect diseases (Fuxa JR, Tanada $Y$ eds) John, Wiley and Sons, New York, 177-214

Carruthers RI, Soper RS (1987) Fungal diseases. In: Epizootiology of insect diseases (Fuxa JR, Tanada Y, eds) John Wiley and Sons, New York, 357-416

Carruthers RI, Robson DS, Roberts DW (1985) In vivo temperature-dependent development of Beauveria bassiana (Deuteromycotina: Hyphomycetes) mycosis of the European corn borer, Ostrinia nubilalis (Lepidoptera: Pyralidae). J Invertebr Pathol 46, 305-311

Deacon JW (1980) Introduction to modern mycology. In: Basic microbiology (Wilkinson JF, ed) vol 7, Univ Edinburgh, $197 \mathrm{p}$

Doberski JW (1981) Comparative laboratory studies on three fungal pathogens of the elm bark beetle Scolytus scolytus: effect of temperature and humidity on infection by Beauveria bassiana, Metarhizium anisopliae and Paecilomyces farinosus. $J$ invertebr Pathol 37, 195-200

Fargues J (1972) Traitement mixte des larves de doryphore Leptinotarsa decemlineata Say par des spores du champignon entomopathogène Beauveria bassiana (Bals) Vuill et des doses réduites d'insecticide. Phytiatr-Phytopharm 21, 170-183

Fargues J (1976) Spécificité des champignons pathogènes imparfaits (hyphomycètes) pour les larves de Coléoptères (Scarabaeidae et Chrysomelidae). Entomophaga 21, 313-323

Fargues J, Rodriguez-Rueda D (1980) Sensibilité des larves de Spodoptera littoralis (Noctuidae) aux hyphomycètes entomopathogènes Nomuraea rileyi et Paecilomyces fumosoroseus. Entomophaga 25, 43-54

Fargues J, Robert PH, Vey A (1976) Rôle du tégument et de la défense cellulaire des Coléoptères hôtes dans la spécificité des souches entomopathogènes de Metarhizium anisopliae (Fungi imperfecti). CR Séances Acad Sci Paris 282, 2223-2226

Ferron P (1967) Étude en laboratoire des conditions écologiques favorisant le développement de la mycose à Beauveria tenella du ver blanc. Entomophaga, 12, 257-293

Ferron $\mathrm{P}$ (1985) Fungal Control. In: Comprehensive insect physiology, biochemistry, and pharmacology (Kerkut GA, Gilbert LI, eds) Pergamon Press, Oxford, vol 12, 313-346 
Ferron P, Hurpin B, Robert PH (1972) Sur la spécificité de Metarhizium anisopliae (Metch) Sor. Entomophaga 17, 165-178

Ferron P, Fargues J, Riba G (1991) Fungi as microbial insecticides against pests. In: Handbook of applied mycology. Humans, animals and insects (Arora DK, Mukerji KG, eds). Marcel Dekker, New York, vol 2, 665-706

Goral VM (1973) Influence de la température sur l'évolution du champignon Beauveria en culture profonde. Zakh Ros/ 17, 48-51 (en russe)

Gray TRG, Williams ST (1971) Soil microrganisms (Heywood VH, ed) Univ Reviews Bot, Edinburgh, $240 p$

Hall RA, Papierok B (1982) Fungi as biological control agents of arthropods of agricultural and medical importance. Parasitology, 84, 205-240

Heinrich B (1981) Insect thermoregulation. John Wiley and Sons, New York, $328 \mathrm{p}$

Ignoffo CM (1981) The fungus Nomuraea rileyi as a microbial insecticide. In: Microbial control of pests and plant diseases, 1970-1980 (Burges HD, ed) Acad Press, New York, 513-538

Ignoffo CM, Puttler B, Hostetter DL, Dickerson WA (1976a) Susceptibility of the cabbage looper, Trichoplusia ni, and velvetbean caterpillar, Anticarsia gemmatalis, to several isolates of the entomopathogenic fungus Nomuraea rileyi. $J$ Invertebr Pathol $28,259-262$

Ignoffo CM, Marston NL, Hostetter DL, Puttler B, Bell JV (1976b) Natural and induced epizootics of Nomuraea rileyi in soybean caterpillars. $J$ Invertebr Pathol 27, 191-198

III'Icheva SN, Alioshina OA, Kononova EA, Yurshenene YAE (1976) Effets de la température sur le développement du Beauveria bassiana (Bals) Vuill, Mikrobiol Fitopatol 10, 87-92 (en russe)

Isaenko NI, Kozachenko VI, Kononova EV, Sarkisova NB, (1974) Influence de la température sur la productivité de différentes souches de Beauveria bassiana. In : Méthode biologique dans la protection des plantes. Thèses et Conférences des Jeunes Chercheurs. Zash Rast, Kichinev, 12-13 (en russe)

Kish LP, Allen GE (1978) The biology and ecology of Nomuraea rileyi and a program for predicting its incidence on Anticarsia gemmatalis in soybean. IFAS Univ Florida, $48 \mathrm{p}$

Maniania NK, Fargues J (1992) Susceptibility of Mamestra brassicae (L), and Spodoptera littoralis (Boisd) larvae (Lep Noctuidae) to the hyphomycetes Paecilomyces fumosoroseus (Brown et
Smith) and Nomuraea rileyi (Samson) at two temperatures. J App/ Entomol 113, 518-524

Messias C, Azevado JL (1980) Parasexuality in the Deuteromycete Metarhizium anisopliae. Trans $\mathrm{Br}$ Mycol Soc 75, 473-477

Mohamed AKA, Sikorowski PP, Bell JV (1977) The susceptibility of Heliothis zea larvae to Nomuraea rileyi at various temperatures. $J$ Invertebr Pathol 21, 444-459

Paris S (1977) Hétérocaryons chez Beauveria tenella. Mycopathologia, 61, 67-75

Poprawski TJ, Marchal M, Robert PH (1985) Comparative susceptibility of Otiorhynchus sulcatus and Sitona lineatus (Coleoptera: Curculionidae) early stages to five entomopathogenic hyphomycetes. Environ Entomol 14, 247-253

Rapilly P (1968) Les techniques de mycologie en pathologie végétale. Ann Epiphyt 19, HS, $102 p$

Riba G, Ravelojoana AM (1984) The parasexual cycle in the entomopathogenic fungus Paecilomyces fumosoroseus (Wize) Brown and Smith. Can J Microbiol $30,922-926$

Riba G, Marcandier S, Richard G, Larget I (1983) Sensibilité de la pyrale du maïs (Ostrinia nubilalis) (Lep: Pyralidae) aux hyphomycètes entomopathogènes. Entomophaga 28, 55-64

Riba G, Glandard A, Ravelojoana AM, Ferron P (1984) Isolement de recombinés mitotiques stables de type «intermédiaire" chez Metarhizium anisopliae (Metschnikoff) par hybridation de biotypes sauvages. CR Sécances Acad Sci Paris, Ser D 291, 657-660

Robert P, Marchal M (1980) Utilisation des larves de Plutella maculipennis (Lep, Hyponomeutidae) comme insecte test de divers Hyphomycètes entomopathogènes (Fungi imperfecti). Entomophaga $25,83-89$

Roberts DW, Campbell AS (1977) Stability of entomopathogenic fungi. Misc Publ Entomol Soc Am 10, 180

Soares GG Jr, Marchal M, Ferron P (1983) Susceptibility of Otiorhynchus sulcatus (Coleoptera: Curculionidae) larvae to Metarhizium anisopliae and Metarhizium flavoviride (Deuteromycotina: Hyphomycetes) at two different temperatures. Environ Entomol 120, 1886-1890

Yurchento I, Zakharov IA, Levitin MM (1974) Génétique et sélection du champignon entomopathogène Beauveria bassiana (Bals) Vuill. Étude de I'hétérocaryose. Genetika 10, 95-101 (en russe) 